\title{
FP-INJECTIVE SEMIRINGS, SEMIGROUP RINGS AND LEAVITT PATH ALGEBRAS
}

DOI:

10.1080/00927872.2016.1226862

\section{Document Version}

Accepted author manuscript

Link to publication record in Manchester Research Explorer

\section{Citation for published version (APA):}

Johnson, M., \& Nam, T. G. (2017). FP-INJECTIVE SEMIRINGS, SEMIGROUP RINGS AND LEAVITT PATH

ALGEBRAS. Communications in Algebra, 45(5), 1893-1906. https://doi.org/10.1080/00927872.2016.1226862

\section{Published in:}

Communications in Algebra

\section{Citing this paper}

Please note that where the full-text provided on Manchester Research Explorer is the Author Accepted Manuscript or Proof version this may differ from the final Published version. If citing, it is advised that you check and use the publisher's definitive version.

\section{General rights}

Copyright and moral rights for the publications made accessible in the Research Explorer are retained by the authors and/or other copyright owners and it is a condition of accessing publications that users recognise and abide by the legal requirements associated with these rights.

\section{Takedown policy}

If you believe that this document breaches copyright please refer to the University of Manchester's Takedown Procedures [http://man.ac.uk/04Y6Bo] or contact uml.scholarlycommunications@manchester.ac.uk providing relevant details, so we can investigate your claim.

\section{OPEN ACCESS}




\title{
FP-INJECTIVE SEMIRINGS, SEMIGROUP RINGS AND LEAVITT PATH ALGEBRAS
}

\author{
M. JOHNSON AND T. G. NAM
}

\begin{abstract}
In this paper we give characterisations of FP-injective semirings (previously termed "exact" semirings in work of the first author). We provide a basic connection between FP-injective semirings and P-injective semirings, and establish that FP-injectivity of semirings is a Morita invariant property. We show that the analogue of the Faith-Menal conjecture (relating FPinjectivity and self-injectivity for rings satisfying certain chain conditions) does not hold for semirings. We prove that the semigroup ring of a locally finite inverse monoid over an FP-injective ring is FP-injective and give a criterion for the Leavitt path algebra of a finite graph to be FP-injective.
\end{abstract}

\section{INTRODUCTION}

The current paper is a continuation of the investigation of FP-injective (or exact) semirings begun in [20]. We extend some well-known results from the theory of FP-injective rings and modules to the setting of semirings and semimodules. In particular, we characterise FP-injective semimodules (Lemma 3.1 and Proposition 3.3), and demonstrate that the properties of exactness and FP-injectivity do indeed coincide. We give several equivalent characterisations of FP-injective semirings in terms of projective and FP-injective semimodules, and P-injective semirings (Theorem 3.4) and apply this result to show that FP-injectivity is a Morita invariant property of semirings (Theorem 3.6). We also consider the relationship between FP-injectivity and self-injectivity, showing that the class of semirings admits counterexamples to the natural analogue of the Faith-Menal conjecture (see Lemma 3.8), and describing the additively regular semirings over which the properties of injectivity and FP-injectivity for semimodules do coincide (Theorem 3.9).

We then consider FP-injectivity for semigroup rings of certain inverse semigroups. We establish a generalisation of a theorem of Garkusha [8]; namely we show that the semigroup ring of a locally finite inverse monoid over an FPinjective ring is FP-injective (Theorem 4.2). We also provide a criterion to check

2000 Mathematics Subject Classification. 16Y60, 16S99, 16 S34.

Key words and phrases. FP-injective semiring; Exact semiring; Leavitt path algebra.

The research of the second author was supported by Vietnam National Foundation for Science and Technology Development (NAFOSTED). 
FP-injectivity of Leavitt path algebras of finite graphs with coefficients in a commutative ring (Theorem 4.4). Our results illustrate that Leavitt path algebras of finite graphs with coefficients in commutative rings do not provide a suitable hunting ground for counterexamples to the Faith-Menal conjecture mentioned above (Corollary 4.5).

\section{Preliminaries}

For the reader's convenience we begin by recording the necessary basic definitions and notation to be used throughout (see [9] for further reference). We recall that a semiring is an algebra $(S,+, \cdot, 0,1)$ satisfying:

(1) $(S,+, 0)$ is a commutative monoid with identity element 0 ;

(2) $(S, \cdot, 1)$ is a monoid with identity element 1 ;

(3) multiplication distributes over addition from either side;

(4) $0 s=0=s 0$ for all $s \in S$.

We say that a semiring $S$ is: commutative if $(S, \cdot)$ is a commutative monoid; additively idempotent if $a+a=a$ for all $a \in S$; additively regular if for each $a \in S, a+b+a=a$ for some $b \in S$; additively cancellative if for all $a, b, c \in S$, $a+c=b+c$ implies that $a=b$; a semifield if $S$ is commutative and $(S \backslash\{0\}, \cdot, 1)$ is a group. Aside from fields, two well-known examples of semifields are the additively idempotent two element semiring $\mathbf{B}=\{0,1\}$ known as the Boolean semifield, and the tropical semifield $\mathbb{T}=(\mathbb{R} \cup\{-\infty\}, \vee,+,-\infty, 0)$. Ideals of semirings, and homomorphisms between semirings are defined in the usual way.

A left $S$-semimodule over a semiring $S$ is a commutative monoid $\left(M,+, 0_{M}\right)$ together with a scalar multiplication $(s, m) \mapsto s m$ from $S \times M$ to $M$ satisfying $\left(s s^{\prime}\right) m=s\left(s^{\prime} m\right), s\left(m+m^{\prime}\right)=s m+s m^{\prime},\left(s+s^{\prime}\right) m=s m+s^{\prime} m, 1 m=m$, $s 0_{M}=0_{M}=0 m$ for all $s, s^{\prime} \in S$ and $m, m^{\prime} \in M$. Right semimodules over $S$ are defined dually. Homomorphisms, subsemimodules, free, projective and injective left (right) $S$-semimodules are defined in the standard manner. As usual, a semimodule is said to be finitely generated if and only if it is a homomorphic image of a free semimodule with a finite basis set, and is said to be cyclic if it is a homomorphic image of a free semimodule of rank 1.

We write ${ }_{S} \mathcal{M}$ (respectively $\mathcal{M}_{S}$ ) to denote the category of left (respectively, right) $S$-semimodules. Semirings $T$ and $S$ are said to be Morita equivalent if the semimodule categories ${ }_{T} \mathcal{M}$ and ${ }_{S} \mathcal{M}$ are equivalent, i.e., there exist additive functors $F:{ }_{T} \mathcal{M} \longrightarrow{ }_{S} \mathcal{M}$ and $G:{ }_{S} \mathcal{M} \longrightarrow{ }_{T} \mathcal{M}$ and natural isomorphisms $\eta: G F \longrightarrow I d_{T} \mathcal{M}$ and $\xi: F G \longrightarrow I d_{S} \mathcal{M}$.

For a semiring $S$ and a matrix $A \in M_{m \times n}(S)$, let $\operatorname{Row}(A)$ denote the left $S$-semimodule of all row vectors $\vec{x} \in S^{n}$ that may be written as $\vec{x}=\vec{u} A$ for some row vector $\vec{u} \in S^{m}$. The right $S$-semimodule $\operatorname{Col}(A)$ is defined dually. Let $M$ be a left $S$-semimodule, and $n$ a positive integer. It is clear that each column vector $\vec{x}=\left[\begin{array}{lll}x_{1} & \ldots & x_{n}\end{array}\right]^{T} \in M^{n}$ induces an $S$-homomorphism $\varphi_{x}: S^{n} \longrightarrow M$ defined by $\varphi_{x}(\vec{s})=\vec{s} \vec{x}:=\sum_{i=1}^{n} s_{i} x_{i}$ for all $\vec{s}=\left[s_{1} \ldots s_{n}\right] \in S^{n}$. We denote by 
$\operatorname{ker}_{S^{n}}^{l}(\vec{x})$ the kernel congruence of $\varphi_{x}\left(m \equiv_{\varphi_{x}} m^{\prime}\right.$ if and only if $\left.\varphi_{x}(m)=\varphi_{x}\left(m^{\prime}\right)\right)$. In particular, if the semimodule $M$ is the free left $S$-semimodule $S^{m}$ for some $m>0$, then $M_{n \times m}(S) \cong\left(S^{m}\right)^{n}$ as left $S$-semimodules. Hence each matrix $A \in M_{n \times m}(S)$ induces an $S$-homomorphism $\varphi_{A}: S^{n} \longrightarrow S^{m}$, and we shall denote the corresponding kernel congruence by $\operatorname{ker}_{S^{n}}^{l}(A)$. If $n=m=1$, then $A=a \in S$, and so $\operatorname{ker}_{S}^{l}(a)$ is determined by $\{(x, y) \in S \times S \mid x a=y a\}$.

\section{FP-INJECTIVE SEMIRINGS}

FP-injectivity and exactness. In [20] Widing, Kambites and the first author introduced exact semirings, defined in terms of certain linear separation (or equivalently, by [20, Theorem 3.4] extension) properties: A semiring $S$ is exact if for every matrix $A$ over $S$, (i) any row vector $\vec{x}$ not contained in $\operatorname{Row}(A)$ can be separated from $\operatorname{Row}(A)$ by column vectors $\vec{t}$ and $\vec{u}$ satisfying $A \vec{t}=A \vec{u}$, but $\vec{x} \vec{t} \neq \vec{x} \vec{u}$ (or equivalently, every $S$-homomorphism from $\operatorname{Row}(A)$ to ${ }_{S} S$ extends to a $S$-homomorphism from $S^{n}$ to ${ }_{S} S$ ); and (ii) any column vector $\vec{y}$ not contained in $\operatorname{Col}(A)$ can be separated from $\operatorname{Col}(A)$ by row vectors $\vec{v}$ and $\vec{w}$ satisfying $\vec{v} A=\vec{w} A$, but $\vec{v} \vec{y} \neq \vec{w} \vec{y}$ (or equivalently, every $S$-homomorphism from $\operatorname{Col}(A)$ to $S_{S}$ extends to a $S$-homomorphism from $S^{m}$ to $S_{S}$ ). By noting that a left (respectively, right) subsemimodule $X \subseteq S^{n}$ is finitely generated if and only if $X=\operatorname{Row}(A)$ (respectively, $X=\operatorname{Col}(A)$ ) for some matrix $A$ over $S$, it is clear that this notion of exactness may be naturally separated into notions of left and right exactness. We begin by comparing left exactness with three related definitions, which are well-known in the context of ring theory (see [16], for example):

(E) A semiring $S$ is called left exact if every $S$-homomorphism $f: X \longrightarrow S$ from a finitely generated left subsemimodule $X \subseteq S^{n}$, can be extended to $S^{n}$.

(S) A semiring $S$ is called left self-injective if the regular left $S$-semimodule $S$ is injective.

(P) A left $S$-semimodule $M$ is $P$-injective if every $S$-homomorphism $f: I \longrightarrow$ $M$ from a principal left ideal $I$ of $S$ can be extended to $S$. A semiring $S$ is left P-injective if the regular left $S$-semimodule $S$ is P-injective.

(FP) A left $S$-semimodule $M$ is $F P$-injective if every $S$-homomorphism $f$ : $X \longrightarrow M$, from a finitely generated left subsemimodule $X$ of a free $S$ semimodule $F$ can be extended to $F$. A semiring $S$ is left FP-injective if the regular left $S$-semimodule $S$ is FP-injective.

Consideration of right semimodules leads to dual notions of right (exact, selfinjective, P-injective, FP-injective) semirings. When $S$ is commutative these notions coincide, and we drop the left/right prefix. These properties are not leftright symmetric in general - see [16, Example 5.46] for the case of FP-injectivity. 
It is immediate from the above definitions that every left self-injective or left FP-injective semiring is left exact, and that every left exact semiring is left Pinjective. Exactness and FP-injectivity are in fact equivalent:

Lemma 3.1. Let $S$ be a semiring and $M$ a left $S$-semimodule. Then $M$ is FPinjective if and only if for each finitely generated subsemimodule $X$ of a finitely generated free left $S$-semimodule $F$, every $S$-homomorphism $X \longrightarrow M$ extends to an $S$-homomorphism $F \longrightarrow M$. In particular, a semiring $S$ is left exact if and only if it is left FP-injective.

Proof. The second statement follows immediately from the first, by noting that the only difference between the definitions $(\mathrm{FP})$ and $(\mathrm{E})$ is that the latter requires extension to finitely generated free semimodules only. The direct implication of the first statement is also clear. For the converse, suppose that $F$ is a free left $S$-semimodule with basis $I$. Since each element of $F$ has finite support, it follows that for any finitely generated subsemimodule $X$ of $F$, there exists a finite subset of $I$ such that every generator of $X$ may be written in terms of this finite subset. Thus there exist free subsemimodules $F_{1}$ and $F_{2}$ of $F$ such that $F=F_{1} \oplus F_{2}, F_{1}$ is finitely generated and $X \subseteq F_{1}$.

Further characterisations of FP-injectivity. Below we give several equivalent characterisations of FP-injective semirings, some of which are the semiring analogues of results for FP-injective rings (see [16, Chapter 5]).

Lemma 3.2 (cf. [16, Lemma 5.1]). For a semiring $S$, the following conditions are equivalent:

(i) $S$ is left P-injective;

(ii) $\operatorname{ker}_{S}^{l}(a) \subseteq \operatorname{ker}_{S}^{l}(b)$, where $a, b \in S$, implies that $b S \subseteq a S$;

(iii) If $\alpha: S a \longrightarrow S, a \in S$, is an $S$-homomorphism of left semimodules, then $\alpha(a) \in a S$.

Proof. Suppose that (i) holds and that $\operatorname{ker}_{S}^{l}(a) \subseteq \operatorname{ker}_{S}^{l}(b)$, where $a, b \in S$. In this case the map $\alpha: S a \longrightarrow S$ given by $\alpha(x a)=x b$ is a well defined $S$-homomorphism and since $S$ is left P-injective, there exists an $S$-homomorphism $\beta: S \longrightarrow S$ such that $\beta(x a)=\alpha(x a)=x b$. But then $b=\beta(a)=a \beta(1) \in a S$, from which it follows that (ii) holds. That (ii) implies (iii) follows immediately from the fact that if $\alpha: S a \longrightarrow S$ is an $S$-homomorphism of left semimodules with $b:=\alpha(a)$, then $\operatorname{ker}_{S}^{l}(a) \subseteq \operatorname{ker}_{S}^{l}(b)$. Finally, suppose that (iii) holds and let $\alpha: S a \longrightarrow S$. By our hypothesis, we may write $\alpha(a)=a c$ for some $c \in S$. Then, the $S$-homomorphism $\beta: S \longrightarrow S$, defined by $\beta(s)=s c$ extends $\alpha$, hence proving (i).

Proposition 3.3 (cf. [16, Theorem 5.39]). For a semiring $S$ and a left $S$ semimodule $M$, the following conditions are equivalent:

(i) $M$ is FP-injective; 
(ii) For each column vector $\vec{m} \in M^{n}$ and $A \in M_{n}(S)$ satisfying $\operatorname{ker}_{S^{n}}^{l}(A) \subseteq$ $\operatorname{ker}_{S^{n}}^{l}(\vec{m})$, we have $\vec{m}=A \vec{x}$ for some column vector $\vec{x} \in M^{n}$;

(iii) For each column vector $\vec{m} \in M^{n}$ and $A \in M_{n \times m}(S)$ satisfying $\operatorname{ker}_{S^{n}}^{l}(A) \subseteq$ $\operatorname{ker}_{S^{n}}^{l}(\vec{m})$, we have $\vec{m}=A \vec{x}$ for some column vector $\vec{x} \in M^{m}$.

Proof. (i) $\Longrightarrow$ (ii): Let $\operatorname{ker}_{S^{n}}^{l}(A) \subseteq \operatorname{ker}_{S^{n}}^{l}(\vec{m})$ and let $\vec{r}_{i}$ denote the $i$-th row of $A$. Then $S^{n} A=\sum_{i} S r_{i}$ is a finitely generated $S$-subsemimodule of $S^{n}$, and our hypothesis shows that $\alpha: S^{n} A \longrightarrow M$ given by $\alpha(\vec{s} A)=\vec{s} \vec{m}$, for all row vector $\vec{s} \in S^{n}$ is a well-defined $S$-homomorphism. Since $M$ is FP-injective, $\alpha$ extends to an $S$-homomorphism $\beta: S^{n} \longrightarrow M$. Let $\left\{e_{1}, \ldots, e_{n}\right\}$ be the canonical basis of the free $S$-semimodule $S^{n}$. For each row vector $\vec{s} \in S^{n}$, we may write $\vec{s}=\sum_{i=1}^{n} s_{i} e_{i}$, where $s_{i} \in S$, and hence, $\beta(\vec{s})=\beta\left(\sum_{i=1}^{n} s_{i} e_{i}\right)=\sum_{i=1}^{n} s_{i} \beta\left(e_{i}\right)=\vec{s} \vec{x}$, where $\vec{x}=\left[\begin{array}{lll}\beta\left(e_{1}\right) \ldots \beta\left(e_{n}\right)\end{array}\right]^{T} \in M^{n}$. If we write $\vec{m}=\left[\begin{array}{lll}m_{1} & \ldots & m_{n}\end{array}\right]^{T} \in M^{n}$, we get

$$
m_{i}=e_{i} \vec{m}=\alpha\left(e_{i} A\right)=\alpha\left(\overrightarrow{r_{i}}\right)=\beta\left(\overrightarrow{r_{i}}\right)=\overrightarrow{r_{i}} \vec{x},
$$

and therefore, $\vec{m}=\left[\overrightarrow{r_{1}} \vec{x}, \ldots, \overrightarrow{r_{n}} \vec{x}\right]^{T}=A \vec{x}$.

(ii) $\Longrightarrow$ (iii): Let $\operatorname{ker}_{S^{n}}^{l}(A) \subseteq \operatorname{ker}_{S^{n}}^{l}(\vec{m})$, where $A \in M_{n \times m}(S)$ and $\vec{m} \in M^{n}$ is a column vector. If $n=m$ then the statement is trivial. If $n>m$, let $A^{\prime}=\left[\begin{array}{ll}A & 0\end{array}\right] \in M_{n}(S)$. Then $\operatorname{ker}_{S^{n}}^{l}\left(A^{\prime}\right)=\operatorname{ker}_{S^{n}}^{l}(A) \subseteq \operatorname{ker}_{S^{n}}^{l}(\vec{m})$, and hence $\vec{m}=A^{\prime} \vec{y}=\left[\begin{array}{ll}A & 0\end{array}\right]\left[\begin{array}{ll}\vec{x} & \vec{z}\end{array}\right]^{T}=A \vec{x}$ for some column vectors $\vec{y}=\left[\begin{array}{ll}\vec{x} & \vec{z}\end{array}\right]^{T} \in M^{n}$ and $\vec{x} \in M^{m}$. If $m>n$, let $A^{\prime}=\left[\begin{array}{ll}A & 0\end{array}\right]^{T} \in M_{m}(S)$ and $\overrightarrow{m^{\prime}}=[\vec{m} 0]^{T} \in M^{m}$. We then have that $\operatorname{ker}_{S^{m}}^{l}\left(A^{\prime}\right) \subseteq \operatorname{ker}_{S^{m}}^{l}\left(\overrightarrow{m^{\prime}}\right)$ and hence by our hypothesis, there exists a column vector $\vec{x} \in M^{m}$ such that $\overrightarrow{m^{\prime}}=\left[\begin{array}{ll}\vec{m} & 0\end{array}\right]^{T}=A^{\prime} \vec{x}=\left[\begin{array}{ll}A & 0\end{array}\right]^{T}$, that is, $\vec{m}=A \vec{x}$.

(iii) $\Longrightarrow$ (i): Let $X$ be a finitely generated subsemimodule of a free left $S$ semimodule $S^{m}$, and $\alpha: X \longrightarrow M$ an $S$-homomorphism. Then we write $X=\sum_{i=1}^{n} S r_{i}$, for some row vectors $r_{i} \in S^{m}$. Set $m_{i}:=\alpha\left(r_{i}\right) \in M, \vec{m}:=$ $\left[\begin{array}{lll}m_{1} & \ldots & m_{n}\end{array}\right]^{T} \in M^{n}$, and let $A$ be the matrix whose $i$ th row is $r_{i}$. Then $\alpha(\vec{s} A)=$ $\vec{s} \vec{m}$ for all row vectors $\vec{s} \in S^{n}$, and hence, $\operatorname{ker}_{S^{n}}^{l}(A) \subseteq \operatorname{ker}_{S^{n}}^{l}(\vec{m})$. By hypothesis there exists a column vector $\vec{x} \in M^{m}$ such that $\vec{m}=A \vec{x}$. So, $m_{i}=r_{i} \vec{x}$ for all $i$. It follows that $\alpha$ extends to an $S$-homomorphism $\beta: S^{m} \longrightarrow M$ defined by $\beta(\vec{s})=\vec{s} \vec{x}$, for all row vectors $\vec{s} \in S^{m}$.

The following theorem gives homological characterisations of left FP-injective semirings in terms of projective and FP-injective semimodules, and provides a relation between left FP-injective semirings and left P-injective semirings. (We note that the equivalence of parts (i) and (iv) of Theorem 3.4 has appeared in [16, Theorem 5.41] for the ring case.)

Theorem 3.4. For a semiring $S$, the following conditions are equivalent:

(i) $S$ is left FP-injective;

(ii) All finitely generated projective left $S$-semimodules are FP-injective;

(iii) All cyclic projective left $S$-semimodules are FP-injective; 
(iv) $M_{n}(S)$ is a left P-injective semiring for each $n \geq 1$.

Proof. It is clear that (ii) implies (iii) and (iii) implies (i). We shall show that (i) implies (ii) and that (i) and (iv) are equivalent.

(i) $\Longrightarrow$ (ii): Let $P$ be a finitely generated projective left $S$-semimodule, $X$ a finitely generated subsemimodule of a free left $S$-semimodule $F$, and $f: X \longrightarrow P$ a n $S$-homomorphism. Since $P \in\left|{ }_{S} \mathcal{M}\right|$ is finitely generated projective, $P$ is a retract of a free left $S$-semimodule $S^{m}$. Thus there exist $S$-homomorphisms $g: S^{m} \longrightarrow P$ and $h: P \longrightarrow S^{m}$ such that $g h=i d_{P}$. Consider the following diagram:

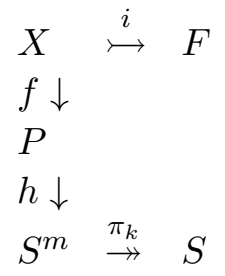

where $\pi_{k}: S^{m} \rightarrow S$ is the canonical $k$-th projection (for all $k=1, \ldots, m$ ), and $i$ is the canonical injection. Since $S$ is a left FP-injective semiring, for each $k$ there exists an $S$-homomorphism $\theta_{k}: F \longrightarrow S$ such that $\theta_{k} i=\pi_{k} h f$. Let us denote by $\iota_{k}: S \longmapsto S^{m}$ the canonical $k$-th injection, and let $\varphi: F \rightarrow P$ be the $S$-homomorphism defined by $\varphi:=g\left(\sum_{k=1}^{m} \iota_{k} \theta_{k}\right)$. We then have that $\varphi i=$ $\sum_{k=1}^{m} g \iota_{k} \theta_{k} i=\sum_{k=1}^{m} g \iota_{k} \pi_{k} h f=g h f=i d_{P} f=f$, or in other words, $\varphi$ extends $f$. It follows that the semimodule $P$ is FP-injective.

$(\mathrm{i}) \Longrightarrow\left(\right.$ iv): Suppose that $\operatorname{ker}_{M_{n}(S)}^{l}(A) \subseteq \operatorname{ker}_{M_{n}(S)}^{l}(B)$, where $A, B \in M_{n}(S)$. We denote by $\overrightarrow{a_{i}}$ and $\overrightarrow{b_{i}}$ the $i$-th columns of the matrices $A$ and $B$, respectively. Then our hypothesis implies that for all $i$,

$$
\operatorname{ker}_{S^{n}}^{l}(A) \subseteq \operatorname{ker}_{S^{n}}^{l}\left(\overrightarrow{b_{i}}\right) .
$$

Since $S$ is left FP-injective, by Proposition 3.3, there exists a column vector $\overrightarrow{x_{i}} \in S^{n}$ such that $\overrightarrow{b_{i}}=A \overrightarrow{x_{i}}$. Setting $X=\left[\overrightarrow{x_{1}} \ldots \overrightarrow{x_{n}}\right] \in M_{n}(S)$, we then have $B=A X$, and so $M_{n}(S)$ is left P-injective by application of Lemma 3.2.

(iv) $\Longrightarrow$ (i): Suppose that $\operatorname{ker}_{S^{n}}^{l}(A) \subseteq \operatorname{ker}_{S^{n}}^{l}(\vec{b})$, where $A \in M_{n}(S)$ and $\vec{b} \in$ $S^{n}$ is a column vector. If $B$ is the matrix with every column equal to $\vec{b}$, then $\operatorname{ker}_{S^{n}}^{l}(A) \subseteq \operatorname{ker}_{S^{n}}^{l}(B)$, and so, $\operatorname{ker}_{M_{n}(S)}^{l}(A) \subseteq \operatorname{ker}_{M_{n}(S)}^{l}(B)$. Since $M_{n}(S)$ is left P-injective, Lemma 3.2 gives $B M_{n}(S) \subseteq A M_{n}(S)$, so in particular $B=A X$ for some matrix $X \in M_{n}(S)$. Hence, $\vec{b}=A \vec{x}$, where $\vec{x}$ is the first column of $X$. Now applying Proposition 3.3 yields that $S$ is FP-injective.

Theorem 3.4 may be applied to prove that direct sums are well behaved with regards to FP-injectivity.

Lemma 3.5. Let $S$ and $R$ be semirings. Then, the semiring $S \oplus R$ is left FPinjective if and only if $S$ and $R$ are both left FP-injective. 
Proof. Assume that $T:=S \oplus R$ is a left FP-injective semiring. We will prove that $S$ and $R$ are left FP-injective. Indeed, let $A \in M_{n}(S)$ and let $\alpha: M_{n}(S) A \longrightarrow$ $M_{n}(S)$ be an $M_{n}(S)$-homomorphism of left semimodules. We first have that $M_{n}(T) \cong M_{n}(R) \oplus M_{n}(S)$, so that any $Z \in M_{n}(T)$ may be written uniquely in the form $Z=X+Y$, where $X \in M_{n}(S)$ and $Y \in M_{n}(R)$. The map $\alpha$ induces an $M_{n}(T)$-homomorphism of left semimodules $\bar{\alpha}: M_{n}(T) A \longrightarrow M_{n}(T)$, defined by $\bar{\alpha}(Z A)=\alpha(X A)$. Since $T$ is left FP-injective, it follows from Theorem 3.4 that $M_{n}(T)$ is left P-injective. Then, by Lemma 3.2, we find that $\bar{\alpha}(A) \in A M_{n}(T)$, and hence $\alpha(A) \in A M_{n}(S)$. From this observation, by applying Lemma 3.2 again we immediately obtain that $M_{n}(S)$ is left P-injective. Since $n$ was arbitrary, it follows that $S$ is left FP-injective, by Theorem 3.4. An identical argument shows that $R$ is a left FP-injective semiring.

For the converse, suppose that $\operatorname{ker}_{M_{n}(T)}^{l}(A) \subseteq \operatorname{ker}_{M_{n}(T)}^{l}(B)$, where $A, B \in$ $M_{n}(S)$. We write $A$ and $B$ in the forms: $A=A_{1}+A_{2}$ and $B=B_{1}+B_{2}$, where $A_{1}, B_{1} \in M_{n}(S)$ and $A_{2}, B_{2} \in M_{n}(R)$. We will show that $\operatorname{ker}_{M_{n}(S)}^{l}\left(A_{1}\right) \subseteq$ $\operatorname{ker}_{M_{n}(S)}^{l}\left(B_{1}\right)$. Indeed, let $X$ and $Y \in M_{n}(S)$ with $X A_{1}=Y A_{1}$. We then have that $X A=Y A$, giving $(X, Y) \in \operatorname{ker}_{M_{n}(T)}^{l}(B)$ and hence $X B_{1}=Y B_{1}$. Similarly, we find that $\operatorname{ker}_{M_{n}(R)}^{l}\left(A_{2}\right) \subseteq \operatorname{ker}_{M_{n}(R)}^{l}\left(B_{2}\right)$, too. From these observations, and the hypothesis that $S, R$ are left FP-injective, we immediately obtain that $B_{1}=$ $A_{1} X$ and $B_{2}=A_{2} Y$ for some $X \in M_{n}(S)$ and $Y \in M_{n}(R)$, by Theorem 3.4 and Lemma 3.2. Hence, $B=B_{1}+B_{2}=A_{1} X+A_{2} Y=A(X+Y)$, so $B \in A M_{n}(T)$. By Lemma 3.2, $M_{n}(T)$ is left P-injective, and applying Theorem 3.4, we get that $T$ is left FP-injective.

Morita invariance. Theorem 3.4 also allows us to show that FP-injectivity is a Morita invariant property of semirings.

Theorem 3.6. Let $F:{ }_{T} \mathcal{M} \rightleftarrows{ }_{S} \mathcal{M}: G$ be an equivalence of the semimodule categories ${ }_{T} \mathcal{M}$ and ${ }_{S} \mathcal{M}$. Then, a semimodule $\left.M \in\right|_{T} \mathcal{M} \mid$ is FP-injective (finitely generated and projective) if and only if $\left.F(M) \in\right|_{S} \mathcal{M} \mid$ is FP-injective (finitely generated and projective). In particular, FP-injectivity is a Morita invariant property of semirings.

Proof. By [14, Proposition 4.8] and [14, Lemma 4.10], we obtain that $\left.M \in\right|_{T} \mathcal{M} \mid$ is finitely generated and projective if and only if $F(M) \in\left|{ }_{S} \mathcal{M}\right|$ is finitely generated and projective, respectively.

Now, let $M$ be an FP-injective left $T$-semimodule. We will prove that $F(M) \in$ $\left|{ }_{S} \mathcal{M}\right|$ is FP-injective too. Let $X$ be a finitely generated subsemimodule of a free left $S$-semimodule $S^{n}$, and $f: X \longrightarrow F(M)$ an $S$-homomorphism, giving the following diagram:

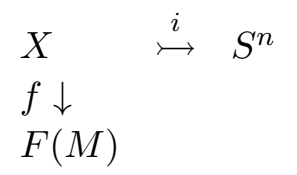


where $i$ is the canonical injection. Applying the functor $G$ to this diagram gives:

$$
\begin{aligned}
& G(X) \quad \stackrel{G(i)}{\longmapsto} G\left(S^{n}\right) \\
& G(f) \downarrow \\
& G(F(M)) \cong M
\end{aligned}
$$

where $G(i)$ is an injective $T$-homomorphism, by the dual of [14, Lemma 4.7]. By the above observation, we may consider $G(X)$ to be a finitely generated subsemimodule of the finitely generated projective left $T$-semimodule $G\left(S^{n}\right)$. Since $\left.G\left(S^{n}\right) \in\right|_{T} \mathcal{M} \mid$ is finitely generated projective, $G\left(S^{n}\right)$ is a retract of a free left $T$-semimodule $T^{m}$. Thus there exist $T$-homomorphisms $\alpha: T^{m} \longrightarrow G\left(S^{n}\right)$ and $\beta: G\left(S^{n}\right) \longrightarrow T^{m}$ such that $\alpha \beta=i d_{G\left(S^{n}\right)}$. Since $\beta$ is injective, $\beta G(i): G(X) \longrightarrow$ $T^{m}$ is injective too. Then, since $M \in\left|{ }_{T} \mathcal{M}\right|$ is FP-injective, there exists a $T$ homomorphism $g: T^{m} \longrightarrow M$ such that $g \beta G(i)=G(f)$, that is, the following diagram commutes.

$$
\begin{aligned}
& G(X) \quad \stackrel{G(i)}{\longmapsto} G\left(S^{n}\right) \stackrel{\beta}{\longmapsto} T^{m} \\
& G(f) \downarrow \quad \downarrow g \\
& M(\cong G(F(M))) \quad=\quad M(\cong G(F(M)))
\end{aligned}
$$

Applying to this diagram the functor $F$, we immediately get that $F(g \beta) i=f$. Therefore, by Lemma 3.1, the $S$-semimodule $F(M)$ is FP-injective. The second statement follows immediately by application of Theorem 3.4.

In [20, Corollary 5.2] it was shown that a matrix semiring $M_{n}(S)$ over an exact (that is, both left and right FP-injective) semiring is exact itself. This result and its converse can be seen as a consequence of the previous theorem.

Corollary 3.7. For a semiring $S$ and a positive integer $n, S$ is left FP-injective if and only if the matrix semiring $M_{n}(S)$ is left FP-injective.

Proof. By [13, Theorem 5.14], $S$ and $M_{n}(S)$ are Morita equivalent semirings. Therefore, using Theorem 3.6, we immediately get the statement.

FP-injectivity and self-injectivity. Although the question posed in [20] of whether there exist exact rings which are not self-injective was quickly resolved (Shitov gave a positive answer to this question in [18], using the fact that a group ring $R G$ is exact if and only if $R$ is exact and $G$ is locally finite, and in fact, this result had previously been obtained by Garkusha [8] phrased in terms of FPinjectivity), there is an interesting related open problem in ring theory concerning the relationship between FP-injectivity and self-injectivity. We recall that a ring is called quasi-Frobenius if it is (left and right) Artinian and self-injective. (There are numerous equivalent conditions characterising quasi-Frobenius rings - see [16] for example). One of the main open problems concerning quasi-Frobenius rings is the so-called Faith-Menal conjecture (see, e.g., [16, pp. xvi]) which states that every right Noetherian and left FP-injective ring is quasi-Frobenius. Recently, 
Shen [17] has shown the Faith-Menal conjecture holds true for rings $R$ for which every closed left ideal of the ring $R$ is finitely generated. At present there are no known counterexamples to this statement. On the other hand, we note that the natural analogue of the Faith-Menal conjecture does not hold for semirings. For instance, the tropical semifield $\mathbb{T}$ provides a counterexample, as we shall now show.

Lemma 3.8. The tropical semifield $\mathbb{T}$ is a left FP-injective semiring that satisfies all chain conditions and yet is not self-injective.

Proof. It is clear that $\mathbb{T}$ satisfies the chain conditions - the only ideals are $\{-\infty\}$ and itself. We note that the results of [20, Section 6] on 'tropical semirings' do not immediately apply to show that $\mathbb{T}$ is FP-injective, since the tropical semirings considered there were anti-involutive, whilst $\mathbb{T}$ is not. However, FP-injectivity of $\mathbb{T}$ can be deduced from FP-injectivity of the extended tropical semiring $\overline{\mathbb{T}}=$ $\mathbb{T} \cup\{\infty\}$ (where the extra element $\infty$ satisfies $s \vee \infty=\infty \vee s=\infty \vee \infty=\infty$ for all $s \in \mathbb{T},(-\infty)+\infty=-\infty$, and $s+\infty=\infty$ for all $-\infty \neq s \in \mathbb{T}) .{ }^{1}$ For the sake of completeness, we give the details of this reduction here.

To prove that $\mathbb{T}$ is left FP-injective, given any $m \times n$ matrix $A$ with entries in $\mathbb{T}$ and any vector $\vec{x}=\left(x_{1}, \ldots, x_{n}\right) \in \mathbb{T}^{n}$ which is not contained in the row space of $A$, we need to find vectors $\vec{t}, \vec{u} \in \mathbb{T}^{n}$ satisfying $A \vec{t}=A \vec{u}$ but $\vec{x} \vec{t} \neq \vec{x} \vec{u}$, under tropical matrix multiplication. It is clear that this can be done if $A$ contains only $-\infty$ entries. In fact, we may assume without loss of generality that $A$ does not contain any rows or columns of $-\infty$ entries. Indeed, if $A$ contains a row of $-\infty$ 's then the condition $A \vec{t}=A \vec{u}$ will be trivially satisfied in the corresponding position for any $\vec{t}$ and $\vec{u}$. Therefore, any solution to the corresponding problem for the matrix $A^{\prime}$ in which all $-\infty$ rows have been deleted, together with the vector $\vec{x}$ (which is also not contained in $\operatorname{Row}\left(A^{\prime}\right)$ ) will be a solution to the original problem. On the other hand, if the $i$ th column of $A$ contains only $-\infty$ 's then the $i$ th entries of $\vec{t}$ and $\vec{u}$ will not play any role in satisfying the equation $A \vec{t}=A \vec{u}$. It follows from this that if $x_{i} \neq-\infty$, then a solution can be found by choosing $\vec{t}$ and $\vec{u}$ as two different appropriate scalings of the $i$ th tropical unit vector, whilst if $x_{i}=-\infty$, any solution to the corresponding problem for the matrix $A^{\prime}$ in which the $i$ th column has been deleted, together with the vector $\vec{x}^{\prime}$ obtained by deleting the position $i$ of $\vec{x}$ (note that this vector will not be in Row $\left(A^{\prime}\right)$ ), may be extended to give a solution of the original problem by inserting $-\infty$ 's in the appropriate position.

It follows from the proof of $\left[10\right.$, Theorem 5.1] that choosing $\vec{t}=(-\vec{x})^{T}$ and $\vec{u}=-\left(\left(-(A \vec{t})^{T}\right) A\right)^{T}$ gives a solution with entries from the extended tropical semiring (where $--\infty=\infty$ ). Notice that if $\vec{x}$ contains only real numbers, then this solution also contains only real numbers. (Indeed, if $\vec{x} \in \mathbb{R}^{n}$, then clearly so

\footnotetext{
${ }^{1}$ It is clear that the extended tropical semiring also satisfies the chain conditions, however this semiring turns out to be self-injective and therefore does not give the required counter-example.
} 
is its negative transpose $\vec{t}$. Since $A$ has no rows of $-\infty$ 's, we note that this yields that $A \vec{t}$ and its negative transpose must be contained in $\mathbb{R}^{n}$. Moreover, since $A$ has no columns of $-\infty$ 's, this also yields that $\left(-(A \vec{t})^{T}\right) A$ and its negative transpose $\vec{u}$ must be contained in $\mathbb{R}^{n}$.) Suppose then that $x_{j}=-\infty$ for some $j$. Let $\vec{t}$ be the $j$ th tropical unit vector, so that $A \vec{t}$ is the $j$ th column of $A$, whilst $\vec{x} \vec{t}=-\infty$. Now taking $\vec{u}$ to be the vector with 0 in position $j$ and $-N$ everywhere else, where $N$ is any positive real number greater than all differences between the entries of $A$, we find that $A \vec{u}$ is again the $j$ th column of $A$, whilst $\vec{x} \vec{u} \neq-\infty$.

It remains to show that $\mathbb{T}$ is not self-injective. Consider $\overline{\mathbb{T}}$ as a left $\mathbb{T}$-semimodule. Since $\mathbb{T}$ is a $\mathbb{T}$-subsemimodule of $\overline{\mathbb{T}}$, if $\mathbb{T}$ were injective then there must be a $\mathbb{T}$ homomorphism $\varphi: \overline{\mathbb{T}} \longrightarrow \mathbb{T}$ such that $\left.\varphi\right|_{\mathbb{T}}=i d_{\mathbb{T}}$. But this would imply that $\varphi(\infty) \vee s=\varphi(\infty) \vee \varphi(s)=\varphi(\infty \vee s)=\varphi(\infty)$ for all $s \in \mathbb{T}$, contradicting the fact that $\mathbb{T}$ has no maximal element. So $\mathbb{T}$ is not self-injective.

FP-injectivity and injectivity of semimodules. Notice that every injective semimodule is obviously FP-injective, but the converse is, in general, not true. The following result describes the additively regular semirings over which the properties of injectivity and FP-injectivity for semimodules do coincide.

Theorem 3.9. For an additively regular semiring $S$, the following conditions are equivalent:

(i) Every left FP-injective S-semimodule is injective;

(ii) Every direct sum of injective left $S$-semimodules is injective;

(iii) $S$ is a left Noetherian ring.

Proof. The fact that (ii) implies (iii) follows from [11, Theorem 3.6] and [12, Theorem 4.2]. That (iii) implies (i) is a consequence of the Baer injectivity criterion. Suppose then that every left FP-injective semimodule is injective. It suffices to show that every direct sum of injective left $S$-semimodules is left FPinjective. Let $\left\{M_{i}\right\}_{i \in I}$ be a family of injective left $S$-semimodules, and $f: X \longrightarrow$ $\bigoplus_{i \in I} M_{i}$ an $S$-homomorphism, where $X$ is a finitely generated subsemimodule of a free left $S$-semimodule $F$. Let $\left\{x_{1}, \ldots, x_{n}\right\}$ be a set of generators of the semimodule $X$. Then for each $j=1, \ldots, n$, there exists a finite subset $I_{j} \subseteq I$ such that $f\left(x_{j}\right) \in \bigoplus_{k \in I_{j}} M_{k}$. Seting $J:=\bigcup_{j=1}^{n} I_{j}$ we note that $J$ is a finite subset of $I$, and $f(X) \subseteq \bigoplus_{i \in J} M_{i}$. Hence $f$ can be considered as a homomorphism $X \longrightarrow$ $\bigoplus_{i \in J} M_{i}$. As each $M_{i}$ is injective, and $J$ is finite, $\bigoplus_{i \in J} M_{i}$ is injective. It follows that $f$ can be extended to an $S$-homomorphism from $F$ to $\bigoplus_{i \in J} M_{i} \subseteq \bigoplus_{i \in I} M_{i}$. Therefore, the left $S$-semimodule $\bigoplus_{i \in I} M_{i}$ is FP-injective.

Note that Theorem 3.9 does not hold if we remove the condition that $S$ is additively regular. Take, for example, the semiring $\mathbb{N}$ of all natural numbers. By $[9$, Proposition 17.21], the only injective $\mathbb{N}$-semimodule is $\{0\}$ showing that $\mathbb{N}$ satisfies condition (ii) of Theorem 3.9 above. 
FP-injectivity and regularity. By [5, Theorem 2.2], every (von Neumann) regular semiring $S$ is P-injective. Using the fact that regularity is a Morita invariant property of rings together with the fact that any ring $R$ is Morita equivalent to the matrix ring $M_{n}(R)$, we see that we may apply Theorem 3.4 to conclude that every von Neumann regular ring is (left and right) FP-injective. This argument does not, however, carry over to the setting of semirings. Whilst it is still the case that any semiring $S$ is Morita equivalent to the matrix semiring $M_{n}(S)$ (see [13, Theorem 5.14]), regularity is not a Morita invariant property of semirings. For example, the tropical semiring is clearly von Neumann regular, whilst it is known that the semiring of $n \times n$ matrices over the tropical semiring is not von Neumann regular for all $n>2$ (see for example [6]). In fact, one can construct examples of von Neumann regular semirings which are not FP-injective. For instance, take the additively cancellative semifield of all nonnegative real numbers $\mathbb{R}^{+}$with the usual addition and multiplication. Clearly, $\mathbb{R}^{+}$is regular, but not FP-injective as the following proposition shows:

Proposition 3.10. Let $S$ be an additively cancellative semifield. Then $S$ is an FP-injective semiring if and only if $S$ is a field.

Proof. Since fields are FP-injective, it remains to show that every additively cancellative FP-injective semifield is a field. Suppose then for contradiction that $S$ is additively cancellative and FP-injective, but not a field. Cancellativity of $S$ tells us that for each $a \in S$ there is at most one element $b \in S$ such that $a+b=0$. Since $S$ is not a field, non-zero elements cannot sum to zero in $S$. (Suppose $a+b=0$ for some non-zero elements $a$ and $b$. It follows that $1+a^{-1} b=0$ and hence $x+x a^{-1} b=0$ for all $x \in S$, giving each element $x \in S$ a unique additive inverse.) Since $S$ is FP-injective, it follows from Theorem 3.4 that the matrix semiring $M_{2}(S)$ is P-injective. Let $A:=\left(\begin{array}{ll}1 & 1 \\ 0 & 1\end{array}\right) \in M_{2}(S)$. We have that for any $X:=\left(\begin{array}{ll}a & b \\ c & d\end{array}\right) \in M_{2}(S), X A=\left(\begin{array}{ll}a & a+b \\ c & c+d\end{array}\right) \in M_{2}(S)$. From this and the fact that the semifield $S$ is additively cancellative, we note that for any $X, Y \in M_{2}(S)$ we have $X A=Y A$ implies that $X=Y$. Thus the map $\alpha: M_{2}(S) A \longrightarrow M_{2}(S)$, given by $\alpha(X A)=X$ for all $X \in M_{2}(S)$ is well defined. Since $M_{2}(S)$ is P-injective and $\alpha$ is an $M_{2}(S)$-homomorphism of left semimodules, applying Lemma 3.2 gives that $\alpha(A) \in A M_{2}(S)$. In other words, there exists a element $Y \in M_{2}(S)$ such that $A Y$ is the identity matrix. Setting $Y:=\left(\begin{array}{cc}x & y \\ z & t\end{array}\right)$ gives $x=1, z=0, t=1, y+t=0$. But since $S$ is zero-sum-free, the last equation simplifies to give $y=0=t$, contradicting $t=1$. 


\section{FP-INJECTIVE INVERSE SEMIGROUP RINGS}

Inverse semigroup rings. The semigroup ring of a semigroup $S$ over a ring $R$ is denoted $R[S]$. Each element of $R[S]$ can be written uniquely as a finite sum $\sum_{s \in S} r_{s} s$ with $r_{s} \in R$. Addition is componentwise and multiplication is determined by the rule $\left(r_{s} s\right)\left(r_{t} t\right)=\left(r_{s} r_{t}\right)(s t)$, where $s, t \in S$ and $r_{s}, r_{t} \in R$. If the semigroup $S$ contains the zero element $\theta$, then $R \theta$ is an ideal of $R[S]$, and the factor ring $R_{0}[S]:=R[S] / R \theta$ is called the contracted semigroup ring of $R$ over $S$. Since $R[S] \cong R_{0}[S] \oplus R \theta$ as a ring direct sum, it follows from Lemma 3.5 that $R[S]$ is left FP-injective if and only if $R_{0}[S]$ is left FP-injective. For this reason $R[S]$ will always, in this note, be understood to be its contracted semigroup ring.

The matrix ring $M_{n}(R)$ may be considered to be the contracted semigroup $\operatorname{ring} R[S]$, where the semigroup $S$ is defined by $S=\left\{e_{i j} \mid 1 \leq i, j \leq n\right\} \cup\{\theta\}$ with $e_{i j} e_{k l}=\theta$ if $j \neq k$ and $e_{i j} e_{k l}=e_{i l}$ if $j=k$. Here $S$ is a (finite) inverse semigroup (every $s \in S$ has a unique "inverse" element $s^{-1}$, satisfying $s s^{-1} s=s$ and $\left.s^{-1} s s^{-1}=s^{-1}\right)$. In light of this fact and Corollary 3.7, it is natural to consider the problem of whether the semigroup ring of an inverse semigroup over a left FP-injective ring is left FP-injective. To this end, we recall that every finite inverse semigroup $S$ has principal series in which the principal factors are isomorphic to Brandt semigroups $B(G, n)$, consisting of all $n \times n$-matrices $(g)_{i j}$, where $g \in G \cup\{\theta\}, G$ a group and $(g)_{i j}$ denotes the matrix with $g$ in the $(i, j)$ th position and the zero element $\theta$ elsewhere, under the natural multiplication, where the underlying groups are the maximal subgroups of $S$.

Lemma 4.1. Let $R$ be a ring and $S$ a finite inverse semigroup. Then $R[S]$ is left FP-injective if and only if $R$ is left FP-injective.

Proof. We first note that by [21, Theorem 2], the semigroup ring $R[S]$ has an identity, say 1. Let

$$
S=S_{0} \supset S_{1} \supset \ldots \supset S_{n+1}
$$

be a principal series for $S$ with $S_{n+1}=\{0\}$ if $S$ has a zero and $S_{n+1}$ empty otherwise. The Rees factor semigroup $S_{i} / S_{i+1}$ is isomorphic to $B(G, m)$, for each $i=0,1, \ldots, n$ (see, e.g., [7, Exercise 3, p. 103]). The proof is by induction on $n$. If $n=0, S \cong S_{0} / S_{1} \cong B(G, m)$, giving $R[S] \cong M_{m}(R[G])$ (see, e.g., [7, Lemma 5.17]), and hence, $R[S]$ is left FP-injective if and only if so is $R[G]$, by Corollary 3.7. Since $G$ is finite, $R[G]$ is left FP-injective if and only if $R$ is left FP-injective (see [8, Theorem 3.2] for example).

Now suppose that that $n>0$. By induction we may assume that $R\left[S / S_{n}\right]$ is left FP-injective if and only if so is $R$, since $S / S_{n}$ is finite inverse semigroup and has a principal series of length less than $n$. Now since $S_{n}$ is isomorphic to $B(G, m)$, we note that $R\left[S_{n}\right]$ has an identity, say $e$. If $x \in R[S]$, then $x e, e x \in R\left[S_{n}\right]$, hence, $x e=e(x e)=(e x) e=e x$ and $e$ is central in $R[S]$, giving the decomposition $R[S]=R[S] e \oplus R[S](1-e)$. Noting that $R[S] e=R\left[S_{n}\right]$ we have $R[S](1-e) \cong$ $R[S] / R\left[S_{n}\right] \cong R\left[S / S_{n}\right]$. Applying Lemma 3.5, $R[S]$ is left FP-injective if and only 
if so are $R\left[S / S_{n}\right]$ and $R\left[S_{n}\right]$. Our inductive hypothesis together with argument used in the case $n=0$ now apply to give the desired result.

Recall that an inverse semigroup $S$ is called locally finite if any finite subset of $S$ generates a finite inverse subsemigroup in $S$. We shall use Lemma 4.1 to prove a generalisation of a result of Garkusha [8, Theorem 3.2], which states that the group ring $R[G]$ is left FP-injective if and only if the ring $R$ is left FP-injective and the group $G$ is locally finite (see also [18, Theorem 3.5]).

Theorem 4.2. Let $R$ be a ring and $S$ a locally finite inverse monoid. Then $R[S]$ is left FP-injective if and only if $R$ is left FP-injective.

Proof. The "only if" part is done similarly as in the proof of [18, Lemma 3.4]. Conversely, for any finite subset $X \subset R[S]$, the union $J$ of all the supports of elements from $X$ is a finite set. By the assumption of the theorem, $J$ generates a finite inverse submonoid $S_{0}$ in $S$, so the subring $R\left[S_{0}\right]$ of $R[S]$ is left FP-injective by Lemma 4.1. Since $X$ is contained in $R\left[S_{0}\right]$, the result follows from [18, Lemma 2.1].

Comparing the previous statement with Garkusha's result, one is drawn to ask whether a semigroup ring $R[S]$ of an inverse monoid (or more generally, monoid) with coefficients in a ring is left FP-injective if and only if $R$ is left FP-injective and $S$ is locally finite. We note that the corresponding statement allowing coefficients from a semiring does not hold since, for example, the semigroup ring $\mathbf{B}[G]$, where $\mathbf{B}$ is the Boolean semifield and $G$ an arbitrary group, is FP-injective, by [20, Example 6.3 and Theorem 6.6].

Cohn path algebras and Leavitt path algebras. We now consider a special class of inverse semigroup rings which are constructed from directed graphs $E=$ $\left(E^{0}, E^{1}, s, r\right)$ with vertex set $E^{0}$ and edge set $E^{1}$, where the maps $s, r: E^{1} \longrightarrow E^{0}$ describe the source and the range of each edge. The graph inverse semigroup $G(E)$ (see for example [15] for further reference) is the semigroup with zero generated by the sets $E^{0}$ and $E^{1}$, together with a set of variables $\left\{e^{*} \mid e \in E^{1}\right\}$, satisfying the following relations for all $v, w \in E^{0}$ and $e, f \in E^{1}$ :

(1) $v w=\delta_{v, w} w$;

(2) $s(e) e=e=e r(e)$ and $r(e) e^{*}=e^{*}=e^{*} s(e)$;

(3) $e^{*} f=\delta_{e, f} r(e)$.

We recall that a path $p=e_{1} \ldots e_{n}$ in the directed graph $E$ is a sequence of edges $e_{1}, \ldots, e_{n}$ such that $r\left(e_{i}\right)=s\left(e_{i+1}\right)$ for $i=1, \ldots, n-1$. In this case we say that the path $p$ starts at $s(p):=s\left(e_{1}\right)$, ends at $r(p):=r\left(e_{n}\right)$ and has length $|p|:=n$. We consider the vertices in $E^{0}$ to be paths of length 0 . A path $p$ of positive length is called a cycle if $s(p)=r(p)$, and $p$ does not revisit any other vertex. We say that the graph $E$ is acyclic if it has no cycles.

For each $v \in E^{0}$, we define $v^{*}:=v$, and for each path $p=e_{1} \ldots e_{n}$ of length $n>0$ we set $p^{*}:=e_{n}^{*} \ldots e_{1}^{*}, r\left(p^{*}\right):=s(p)$ and $s\left(p^{*}\right):=r(p)$. With this notation, 
every nonzero element of $G(E)$ may be written uniquely as $p q^{*}$ for some paths $p, q$ having $r(p)=r(q)$, and $G(E)$ is seen to be an inverse semigroup, with $\left(p q^{*}\right)^{-1}=q p^{*}$ for all paths $p, q$.

From now on let $R$ be a commutative ring and $E$ a finite graph. The (contracted) semigroup ring $R[G(E)]$ is known as the Cohn path algebra of $E$ with coefficients in $R$, denoted by $C_{R}(E)$. We say that a vertex $v$ of $E$ is a sink if $s^{-1}(v)$ is empty. Let $I$ be the ideal of $C_{R}(E)$ generated by all elements of the form $v-\sum_{e \in s^{-1}(v)} e e^{*}$, where $v$ is a non-sink vertex. The $R$-algebra $C_{R}(E) / I$ is easily seen to be the Leavitt path algebra of $E$ with coefficients in $R$ (see [1], [19] for further reference). Since $E$ is finite, both $C_{R}(E)$ and $L_{R}(E)$ are unital rings, each having identity $1=\sum_{v \in E^{0}} v$ (see [2, Subsection 4.2] for example). Furthermore, in [19, Proposition 4.7] Tomforde has shown that one can obtain a $\mathbb{Z}$-grading on the Leavitt path algebra $L_{R}(E)$ by setting:

$$
L_{R}(E)_{n}:=\left\{\sum_{k=1}^{l} r_{k} \alpha_{k} \beta_{k}^{*} \mid r_{k} \in R, \alpha_{k}, \beta_{k} \text { are paths and }\left|\alpha_{k}\right|-\left|\beta_{k}\right|=n\right\}
$$

The structure of the Leavitt path algebra of a finite acyclic graph $E$ with coefficients in a field $K$ is given in [2, Proposition 3.5]. Repeating verbatim the proofs of [2, Lemma 3.4 and Proposition 3.5], we note that the result extends to the case of Leavitt path algebras with coefficients in a commutative ring:

Lemma 4.3 (cf. [2, Proposition 3.5]). Let $E$ be a finite acyclic graph, $R$ a commutative ring, and $\left\{v_{1}, \ldots, v_{k}\right\}$ the set of sinks. Then $L_{R}(E) \cong \bigoplus_{i=1}^{k} M_{n\left(v_{i}\right)}(R)$, where for each $i=1, \ldots, k, n\left(v_{i}\right)$ is the number of paths ending in the sink $v_{i}$.

For a finite acyclic graph $E$ it is easy to see that the graph inverse semigroup $G(E)$ is finite, and hence, by Lemma 4.1, the Cohn path algebra $C_{R}(E)$ is left FP-injective if and only if the commutative ring $R$ is an FP-injective ring. Furthermore, in [3, Theorem 1.5.17] Abrams et. al. showed a perhaps-surprising connection between Cohn and Leavitt path algebras in that every Cohn path algebra is, in fact, a Leavitt path algebra. (Although they expressed the result for Cohn path algebras with coefficients in fields, one can extend easily it to Cohn path algebras with coefficients in commutative rings.) In light of these results, it is therefore natural to ask if there exist left FP-injective Leavitt path algebras or Cohn path algebras where the underlying graph contains cycles.

Theorem 4.4. Let $E$ be a finite graph and $R$ a commutative ring. Then the following conditions are equivalent:

(i) $L_{R}(E)$ is left FP-injective;

(ii) $E$ is acyclic and $R$ is FP-injective;

(iii) $R$ is FP-injective and $L_{R}(E) \cong \bigoplus_{i=1}^{k} M_{n\left(v_{i}\right)}(R)$, where $k$ is the number of sinks in $E$ (call them $v_{1}, \ldots, v_{k}$ ), and for each $i=1, \ldots, k, n\left(v_{i}\right)$ is the number of paths ending in the sink $v_{i}$;

(iv) $L_{R}(E)$ is right FP-injective. 
Proof. (i) $\Longrightarrow$ (ii): Assume that $L_{R}(E)$ is left FP-injective. We first prove that $E$ is acyclic. Indeed, suppose $E$ is not acyclic, that is, $E$ contains a cycle $c$, and let $v:=s(c)=r(c)$. We note that $L_{R}(E)$ has identity $1=\sum_{u \in E^{0}} u$ (see, e.g., [2, Subsection 4.2]), and $c v=c=v c$. It follows from our hypothesis that $L_{R}(E)$ is left $P$-injective, and we note that any element of a left P-injective semiring has a right inverse if and only if it is left cancellative (The "only if" part is true without any assumption on $S$. Conversely, suppose $s \in S$ is left cancellative. Thus whenever $x, y \in S$ satisfy $x s=y s$, we have that $x=y$, or in other words, $\operatorname{ker}_{R}^{l}(s) \subseteq \operatorname{ker}_{R}^{l}(1)$. Since $S$ is left P-injective we may apply Lemma 3.2 to find that $S \subseteq s S$, and in particular, $1=s r$ for some element $r \in S$ ). It then follows that $1-c$ either has a right inverse, or is a right zero-divisor in $L_{R}(E)$. Thus $1-c$ has a right inverse, there exists an element $\alpha \in L_{R}(E)$ such that $(1-c) \alpha=1$. It follows that $(v-c) \alpha(v-c)=v-c$. Since $(v-c) v=v-c=v(v-c)$, we have $(v-c) v \alpha v(v-c)=v-c$, hence, we may assume that $\alpha=v \alpha v$. By [2, Proposition 4.7], we write $\alpha=\sum_{i=m}^{n} a_{i}$ as a graded sum of homogeneous elements, where $a_{m} \neq 0, a_{n} \neq 0, \operatorname{deg}\left(a_{i}\right)=i$ for all nonzero $a_{i}$ having $m \leq i \leq n$. Then, by substituting for $\alpha$ in the equation above, we get the equation

$$
(v-c)\left(\sum_{i=m}^{n} a_{i}\right)(v-c)=v-c .
$$

To reach a contradiction, we essentially follow the ideas in the proof of $(2) \Longrightarrow(3)$ in $[4$, Theorem 1$]$, by expanding the above equation, considering it as a graded equation and comparing the degree of the components on both sides. Existence of terms of higher degrees on the left hand side with no terms of equal degree on the right hand side leads to a contradiction. Hence, $1-c$ is a right zerodivisor in $L_{K}(E)$, that is, $\beta(1-c)=0$ for some nonzero element $\beta \in L_{K}(E)$, or equivalently, $\beta=\beta c$. But this is impossible, by using the above note and comparing the degree of the components on both sides. Thus $E$ is acyclic.

Let $\left\{v_{1}, \ldots, v_{k}\right\}$ be the set of all sinks in $E$, and $n\left(v_{i}\right)$ the number of paths ending in the sink $v_{i}$. By Lemma $4.3, L_{R}(E) \cong \bigoplus_{i=1}^{k} M_{n\left(v_{i}\right)}(R)$. Now, using Lemma 3.5, we obtain that $M_{n\left(v_{i}\right)}(R)$ is left FP-injective for each $i$. Applying Corollary 3.7, we immediately get that $R$ is FP-injective.

That (ii) implies (iii) follows from Lemmas 3.5 and 4.3, and Corollary 3.7. That (iii)implies (i) follows from Corollary 3.7 and Lemma 3.5. The remaining proofs are done similarly.

It follows from Theorem 4.4 that a counterexample to the Faith-Menal conjecture cannot be found in the class of the Leavitt path algebras of finite graphs with coefficients in commutative rings:

Corollary 4.5. Let $E$ be a finite graph and $R$ a commutative ring. Then the following conditions are equivalent:

(i) $L_{R}(E)$ is Noetherian and FP-injective; 
(ii) $L_{R}(E)$ is right Noetherian and left FP-injective;

(iii) $R$ is quasi-Frobenius and $L_{R}(E) \cong \bigoplus_{i=1}^{k} M_{n\left(v_{i}\right)}(R)$, where $k$ is the number of sinks in $E$ (call them $\left.v_{1}, \ldots, v_{k}\right)$, and for each $i=1, \ldots, k, n\left(v_{i}\right)$ is the number of paths ending in the sink $v_{i}$.

Furthermore, if the above conditions are satisfied then $L_{R}(E)$ is a quasi-Frobenius ring.

Proof. It is clear that (i) implies (ii). Suppose that (ii) holds. Then by Theorem 4.4, $R$ is FP-injective and $L_{R}(E) \cong \bigoplus_{i=1}^{k} M_{n\left(v_{i}\right)}(R)$, where $k$ is the number of sinks in $E$ (call them $v_{1}, \ldots, v_{k}$ ), and for each $i=1, \ldots, k, n\left(v_{i}\right)$ is the number of paths ending in the sink $v_{i}$. Since $L_{R}(E)$ is right Noetherian, $M_{n\left(v_{i}\right)}(R)$ is also right Noetherian for each $i=1, \ldots, k$. It follows that $R$ is Noetherian. From this observation, the fact that $R$ is FP-injective and the Baer criterion for injectivity, it is immediate that $R$ is a self-injective ring. Then, by [16, Theorem 1.50], $R$ is quasi-Frobenius, giving statement (iii).

Finally, suppose that (iii) holds. Since $R$ is quasi-Frobenius, so are $M_{n\left(v_{i}\right)}(R)$ for all $i=1, \ldots, k$, and hence, $L_{R}(E)$ is a quasi-Frobenius ring, too. Applying [16, Theorem 1.50] now gives (i).

Regularity conditions for Leavitt path algebras. Leavitt path algebras of finite acyclic graphs over fields have extremely nice properties. Indeed, combining Theorem 4.4 above and [4, Theorem 1] gives the following result:

Corollary 4.6. Let $E$ be a finite graph and $K$ a field. Then the following conditions are equivalent:

(i) $E$ is acyclic;

(ii) $L_{K}(E)$ is left FP-injective;

(iii) $L_{K}(E)$ is left P-injective;

(iv) $L_{K}(E) \cong \bigoplus_{i=1}^{k} M_{n\left(v_{i}\right)}(K)$, where $k$ is the number of sinks in $E$ (call them $\left.v_{1}, \ldots, v_{k}\right)$, and for each $i=1, \ldots, k, n\left(v_{i}\right)$ is the number of paths ending in the sink $v_{i}$;

(v) $L_{K}(E)$ is (von Neumann) regular;

(vi) $L_{K}(E)$ is $\pi$-regular;

(vii) $L_{K}(E)$ is strongly $\pi$-regular.

The condition that $K$ be a field in the previous corollary cannot be weakened to allow any commutative FP-injective ring. For example, taking $R=\mathbb{Z} / 4 \mathbb{Z}$ and $E$ to be the graph with one vertex and no edges yields that the Leavitt path algebra $L_{R}(E) \cong R$ is both FP-injective and $\pi$-regular, but not regular. However, using the proofs of Theorem 4.4, Corollary 4.5 and [4, Theorem 1], we get the following facts concerning the regularity of more general Leavitt path algebras:

Proposition 4.7. Let $E$ be a finite graph and $R$ a commutative ring. Then the following conditions are equivalent: 
(i) $L_{R}(E)$ is (von Neumann) regular;

(ii) $R$ is regular and $E$ is acyclic;

(iii) $R$ is regular and $L_{R}(E) \cong \bigoplus_{i=1}^{k} M_{n\left(v_{i}\right)}(R)$, where $k$ is the number of sinks in $E$ (call them $\left.v_{1}, \ldots, v_{k}\right)$, and for each $i=1, \ldots, k, n\left(v_{i}\right)$ is the number of paths ending in the sink $v_{i}$.

Proof. Using Theorem 4.4, the fact that regularity is a Morita invariant property of rings, and the trivial fact that a finite product of regular rings is regular.

Proposition 4.8. Let $E$ be a finite graph and $R$ a commutative Noetherian ring. Then the following conditions are equivalent:

(i) $L_{R}(E)$ is FP-injective;

(ii) $R$ is quasi-Frobenius and $E$ is acyclic;

(iii) $R$ is quasi-Frobenius and $L_{R}(E) \cong \bigoplus_{i=1}^{k} M_{n\left(v_{i}\right)}(R)$, where $k$ is the number of sinks in $E$ (call them $v_{1}, \ldots, v_{k}$ ), and for each $i=1, \ldots, k, n\left(v_{i}\right)$ is the number of paths ending in the sink $v_{i}$;

(iv) $R$ is quasi-Frobenius and $L_{R}(E)$ is strongly $\pi$-regular.

(v) $R$ is quasi-Frobenius and $L_{R}(E)$ is $\pi$-regular;

Proof. The equivalence of (i), (ii) and (iii) follows immediately from Theorem 4.4.

(iii) $\Longrightarrow$ (iv): By Corollary 4.5, $L_{R}(E)$ is quasi-Frobenius, in particular, it is Artinian. Then, for $a \in L_{R}(E)$, the descending chain of right ideals $a L_{R}(E) \supseteq$ $a^{2} L_{R}(E) \supseteq \ldots \supseteq a^{n} L_{R}(E) \supseteq$.. becomes stationary after finitely many terms. Similarly for the descending chain of left ideals $L_{R}(E) a \supseteq L_{R}(E) a^{2} \supseteq \ldots \supseteq$ $L_{R}(E) a^{n} \supseteq$.. Hence, $L_{R}(E)$ is strongly $\pi$-regular.

(iv) $\Longrightarrow(\mathrm{v})$ : Using again the proof of direction $(5) \Longrightarrow(2)$ of $[4$, Theorem 1$]$.

(v) $\Longrightarrow($ ii): Let $I$ be an arbitrary maximal ideal of $R, K:=R / I$, and $\pi$ : $R \longrightarrow K$ the canonical surjection. Then one has that $K$ is a field, and there is a nonzero surjective ring homomorphism $\varphi: L_{R}(E) \longrightarrow L_{K}(E)$ such that $\varphi\left(r p q^{*}\right)=\pi(r) p q^{*}$, where $r \in R$ and $p, q$ are paths in $E$ and $r(p)=r(q)$. Since $L_{R}(E)$ is $\pi$-regular, so is $L_{K}(E)$. Now applying [4, Theorem 1] yields that $E$ is acyclic.

Acknowledgements. The authors thank Prof. S. N. Il'in (N.I. Lobachevsky Institute of Mathematics and Mechanics, Kazan Federal University) for his helpful discussions with the second author, concerning self-injectivity of the extended tropical semiring $\overline{\mathbb{T}}=\mathbb{R} \cup\{+\infty,-\infty\}$.

\section{REFERENCES}

[1] G. Abrams and G. Aranda Pino. (2005). The Leavitt path algebra of a graph. J. Algebra 293:319-334.

[2] G. Abrams, P. Ara, and M. Siles Molina. (2007). Finite-dimensional Leavitt path algebras. Journal of Pure and Applied Algebra 209:753-762. 
[3] G. Abrams, P. Ara, and M. Siles Molina. Leavitt path algebras. Lecture Notes in Mathematics series, Springer-Verlag Inc. (to appear).

[4] G. Abrams and K. M. Rangaswamy. (2010). Regularity conditions for arbitrary Leavitt path algebras. Algebr. Represent. Theory 13:319-334.

[5] J. Ahsan, M. Shabir and H. J. Weinert. (1998) Characterizations of semirings by P-injective and projective semimodules. Commun. Algebra 26:2199-2209.

[6] G. Cohen, S. Gaubert, J.-P. Quadrat. (1997). Linear projectors in the max-plus algebra. In Proceedings of the 5th IEEE Mediterranean Conference on Control and Systems, Paphos, Cyprus.

[7] A. H. Clifford and G. B. Preston. (1961). Algebraic theory of semigroups. Vol. I, Math. Surveys, No. 7, Amer. Math. Soc., Providence, R. I.

[8] G. Garkusha. (2002). FP-injective and weakly quasi-Frobenius rings. Journal of Mathematical Sciences 112:4303-4312.

[9] J.S. Golan. (1999) Semirings and their Applications. Kluwer Academic Publishers, Dordrecht-Boston-London.

[10] C. Hollings, M. Kambites. (2012). Tropical matrix duality and Green's $\mathcal{D}$ relation. J. Lond. Math. Soc. 86:520-538.

[11] S. N. Il'in. (2010). Direct Sums of Injective Semimodules and Direct Products of Projective Semimodules Over Semirings. Russian Mathematics 54:27-37.

[12] Y. Katsov. (1994). Tensor products and injective enveloples of semimodules over additively regular semirings. Algebra Colloquium 4:121-131.

[13] Y. Katsov. (2004). Toward homological characterization of semirings: Serre's conjecture and Bass's perfectness in a semiring context. Algebra Universalis 52:197-214.

[14] Y. Katsov, T. G. Nam. (2011). Morita Equivalence and Homological Characterization of Semirings. J. Algebra Appl. 10:445-473.

[15] Z. Mesyan and J. D. Mitchell. (2016). The structure of a graph inverse semigroup. Semigroup Forum. DOI 10.1007/s00233-016-9793-x.

[16] W. K. Nicholson and M. F. Yousif. (2003). Quasi-Frobenious rings. Cambridge Univ. Press, Vol. 158.

[17] L. Shen (2016). A note on the Faith-Menal conjecture. Commu. Algebra 44:95-100.

[18] Y. Shitov. (2014). Group rings that are exact. J. Algebra 403:179-184.

[19] M. Tomforde. (2011). Leavitt path algebras with coefficients in a commutative ring. J. Pure Appl. Algebra 215:471-484.

[20] D. Wilding, M. Johnson and M. Kambites. (2013). Exact rings and semirings. J. Algebra 388:324-337.

[21] R. Wenger. (1969). Self-injective semigroup rings for finite inverse semigroups. Proc. Amer. Math. Soc. 20:213-216.

School of Mathematics, University of Manchester, Manchester M13 9PL, UK.

E-mail address: Marianne.Johnson@maths.manchester.ac.uk

Institute of Mathematics, VASt, 18 Hoang Quoc Viet, Cau Giay, Hanoi, Vietnam. E-mail address: tgnam@math.ac.vn 